\title{
A study to assess the association of various factors of adherence to treatment on the tuberculosis treatment outcome among new sputum smear positive pulmonary tuberculosis patients under Revised National Tuberculosis Control Programme in Bengaluru area
}

\author{
Ramya M. S. ${ }^{*}$, Jyothi Jadhav ${ }^{2}$, Ranganath T. S. ${ }^{2}$
}

Department of Community Medicine, ${ }^{1}$ East Point College of Medical Sciences and Research Center, ${ }^{2}$ Bangalore Medical College and Research Institute, Bengaluru, Karnataka, India

Received: 15 July 2019

Revised: 05 September 2019

Accepted: 06 September 2019

\section{*Correspondence:}

Dr. Ramya M. S.,

E-mail: ramyamohan146@gmail.com

Copyright: (c) the author(s), publisher and licensee Medip Academy. This is an open-access article distributed under the terms of the Creative Commons Attribution Non-Commercial License, which permits unrestricted non-commercial use, distribution, and reproduction in any medium, provided the original work is properly cited.

\section{ABSTRACT}

Background: Tuberculosis (TB) is a top infectious disease killer worldwide. In India although Revised National Tuberculosis Control Programme (RNTCP) has seen significant success in TB treatment, patient non-adherence or lost to follow up continue to persist and are influenced by various factors. The present study aims to know the influence of adherence factors on TB treatment outcome among new sputum smear positive (NSSP) pulmonary TB patients under RNTCP. The objectives of the present study were to describe the distribution of socio-demographic factors, outcome patterns among NSSP patients and to assess the association of various adherence factors on TBtreatment outcome among NSSP patients.

Methods: A prospective longitudinal study among 149 NSSP patients from selected TU's of Bengaluru.

Results: Among the 149 NSSP patients 107 (72\%) were males and 42 (28\%) were females. Treatment outcome is categorized as cured (120) and other treatment outcomes (defaulted-17 or treatment failure-3 or died-9). Among the various socio-demographic and adherence-factors: age >50years, illiteracy, male-gender, Hindu-religion, lower socioeconomic-status, poor-patient knowledge about TB, disbelief in TB-treatment, unwillingness to continue treatment with subsiding symptoms, smoking, alcohol intake, presence of diabetes or hypertension or COPD or HIV, patient's un-satisfaction with treatment-availability or accessibility or contact-timings, no-encouragement from family members, other marital status and absence of stigma showed lower cure rates. Of these factors illiteracy, lower socioeconomic-status, poor patient's knowledge on tuberculosis, smoking, alcohol intake, HIV positive status, unsatisfaction with TB-treatment availability and other marital-status showed statistical significance on the TB treatment outcome.

Conclusions: With the observed associations of the above factors on TB-treatment outcome, Further measures like improving patient's knowledge on tuberculosis, health-services and patient-provider relationship; appropriate TBHIV care and encouragement to quit smoking or alcohol intake, could improve TB-treatment cure rates.

Keywords: Pulmonary tuberculosis, Adherence factors, Treatment outcome 


\section{INTRODUCTION}

Tuberculosis caused by Mycobacterium tuberculosis affects the lungs causing pulmonary TB. ${ }^{1}$ TB is a top infectious disease killer worldwide. Ending the TB epidemic by 2030 is among the health targets of the sustainable development goals. ${ }^{2}$ The global plan to end TB 2016-2020 targets 90-90-90 reach 90\% who need TB treatment, including $90 \%$ of people in key populations and achieve $90 \%$ treatment success. ${ }^{3}$ Tuberculosis has a large scale impact on morbidity, mortality and disability. Global incidence of TB is 9.6 million (176/lakh/year) and a TB mortality of 1.1 million (21/lakh/year). In India the incidence of TB is 2.2 million (167/lakh/year) and a TB mortality of 2.2 lakhs (17/lakh/year), were India accounts to about $1 / 4^{\text {th }}$ of the global TB incident cases. ${ }^{5}$ In India under Revised National Tuberculosis Control Programme (RNTCP) new cases of drug-susceptible TB are treated with isoniazid, rifampicin, ethambutol and pyrazinamide. ${ }^{6}$ The treatment success rate is $>85 \%$. $^{7}$ Treatment for drug resistant (DR) TB requires expensive and toxic drugs and treatment success rates are much lower. ${ }^{6}$ Adherence to treatment is defined as the extent to which the patient's therapeutic drug-taking coincides with the prescribed treatment. Poor adherence may lead to incomplete anti-tuberculosis treatment (ATT) which may result in treatment failure, relapse and contribute to increase in duration and cost of treatment, morbidity or mortality and further increasing drug resistance both at individual and community level. ${ }^{8}$ The adherence to TB treatment is influenced by various factors:

\section{Social and economic factors}

TB usually affects people lacking social support networks, unemployed, poor and homeless.

\section{Patient related factors}

Patient's knowledge about TB treatment, perception of benefits from therapy, demographic characteristics such as age, race, gender, education and SES.

\section{Provider characteristics}

Training of health care provider and patient-provider relationship.

\section{Pattern of health care delivery}

Organization of clinical services, expertise, links with patient support system and flexibility in hours of operation. ${ }^{8}$

\section{Characteristics of treatment}

Number of medications, duration or complexity of regimen, toxicity and other side effects.

\section{Disease characteristics}

Non adherence is common with resolution of symptoms following treatment. ${ }^{9}$

In India although RNTCP programme has seen significant success, treatment non-adherence and lost to follow up continue to persist. This increases the risk of conversion of drug sensitive TB to drug resistant TB (rifampicin resistant TB or MDRTB or XDR or TDRTB), further with each progressing levels the chances of survival gets slimmer and cost of medication increases. The association of adherence factors on the TB treatment outcome helps to understand, the role of health services and patient-care in improving the treatment outcome. Thus this study aims at understanding these adherence factors on treatment outcome.

\section{METHODS}

This study is a hospital based (TU's) time bound prospective longitudinal study, after obtaining clearance from the Institutional Ethics Committee and permissions from State and BBMP TB authorities the data collection was started.

By multistage random sampling technique the DOTS center's and study participants were selected in the BBMP (Bruhat Bengaluru Mahanagar Pallike) area as shown in Figure 1.

Adult ( $\geq 18$ years) NSSP pulmonary TB patients (category 1) initiating their treatment in these selected DOTS centres during June to September 2015 and consenting to take part in the study were initially included (162) in the study. The patients were followed up for 6-8 months (upto May 2016) for their treatment outcomes. The patients switching over from DOTS therapy or transferring out of the study area or not evaluated were excluded (13), thus contributing to a total of 149 participants for analysis. New sputum smear positive (NSSP) cases were interviewed by personal interview method during their intensive and continuation phase of DOTS therapy at the DOTS centres or DMC. By using semi-structured, pre-tested proforma, patient's socio-demographic details, data on adherence factors such as patient-related factors, health-care factors, social factors were collected and further followed up for their treatment outcomes. Data was entered into excel-sheet, analyzed using SPSS software 20 version. Sociodemographic data is presented as descriptive statistics; association of adherence factors on the treatment outcome were analyzed as cure rates, risk-ratio or relative-risk, risk-difference or attributable risk, attributable fraction in exposed and statistical significance was interpreted by Chi-square test, $\mathrm{p}$ value. Further data is presented in the form of tables and figures. 


\section{BBMP area with14 RNTCP TU's, 317 DOTS centres}

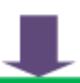

By Simple Random sampling 7 TU's were chosen

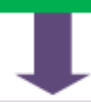

By Simple Random sampling 4 DOTS centers were chosen from each of the 7 TU's

\section{$7 \times 4=28$ DOTS centers were selected.}

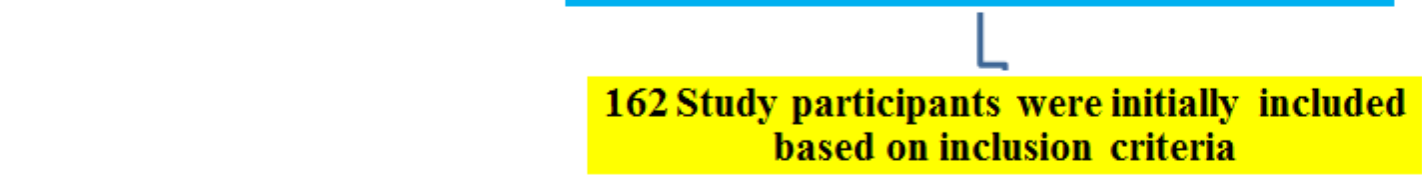

Based on exclusion criteria

13 participants were excluded from analysis

\section{Total of 149 participants were analysed $(\mathbf{N})$}

Figure 1: Sampling of study participants.

\section{RESULTS}

In the present study out of a total of 149 NSSP pulmonary TB patients', age-wise distribution is shown in Table 1.

Table 1: Age wise distribution of the study participants.

\begin{tabular}{|lll|}
\hline Age group & Number & \% \\
\hline $\mathbf{1 8 - 2 0}$ & 12 & 8.05 \\
\hline $\mathbf{2 1 - 3 0}$ & 40 & 26.84 \\
\hline $\mathbf{3 1 - 4 0}$ & 40 & 26.84 \\
\hline $\mathbf{4 1 - 5 0}$ & 33 & 22.14 \\
\hline $\mathbf{5 1 - 6 0}$ & 16 & 10.73 \\
\hline $\mathbf{6 1 - 7 0}$ & 5 & 3.35 \\
\hline $\mathbf{7 0}$ & 3 & 2.01 \\
\hline Total & 149 & \\
\hline
\end{tabular}

Distribution of participants based on gender majority were males $107(71.81 \%)$ and $42(28.18 \%)$ were females. Based on religion the participants were distributed as Hindus 120 (80.53\%), Muslims 27 (18.12\%) and Christians $2(1.34 \%)$. Based on family type majority 102 $(68.45 \%)$ were from nuclear family, $33(22.14 \%)$ from three generation family, $8(5.36 \%)$ were from joint family and $6(4.02 \%)$ were living alone (who had either lost or were separated from their family members). Based on current habits during the study period majority 86
(57.71\%) were smoking tobacco (any form), 55 (36.91\%) of them consumed alcohol and $14(9.39 \%)$ of them chewed tobacco. Based on marital status $94(63.08 \%)$ were married, $41(27.51 \%)$ were unmarried, $12(8.05 \%)$ were separated and $2(1.34 \%)$ were divorced. Distribution of participants based on literacy status is shown in Figure 2 , occupation is shown in Figure 3, socio-economic classification (as per Modified-Kuppuswamy's scale 2015) is shown in Figure 4 and the presence of comorbidities is shown in Figure 5.The treatment outcome pattern among the 149 new sputum smear positive pulmonary tuberculosis patients-120 (80.53\%) were cured, 17 (11.40\%) had defaulted, 3 (2.01\%) had treatment failure and $9(6.04 \%)$ had died during the treatment course.



Figure 2: Distribution of study subjects based on education $(n=149)$. 


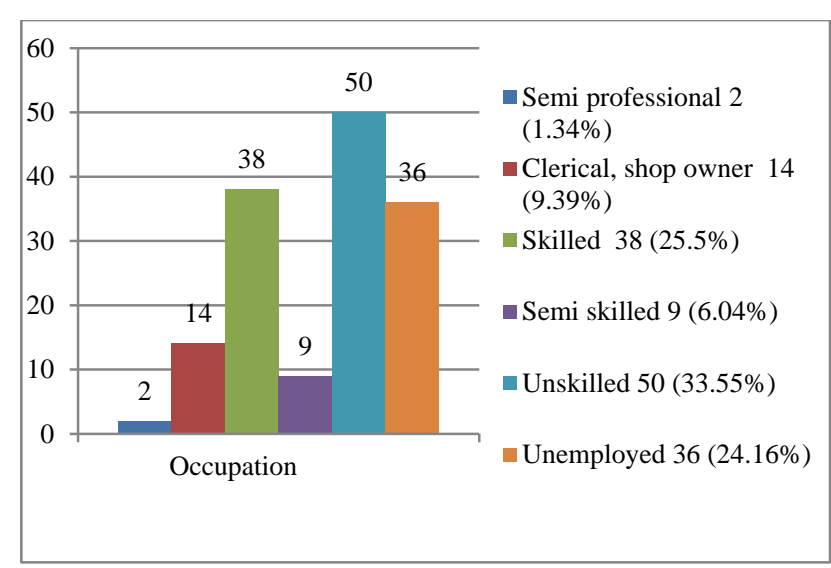

Figure 3: Distribution of study subjects based on their occupation $(n=149)$.

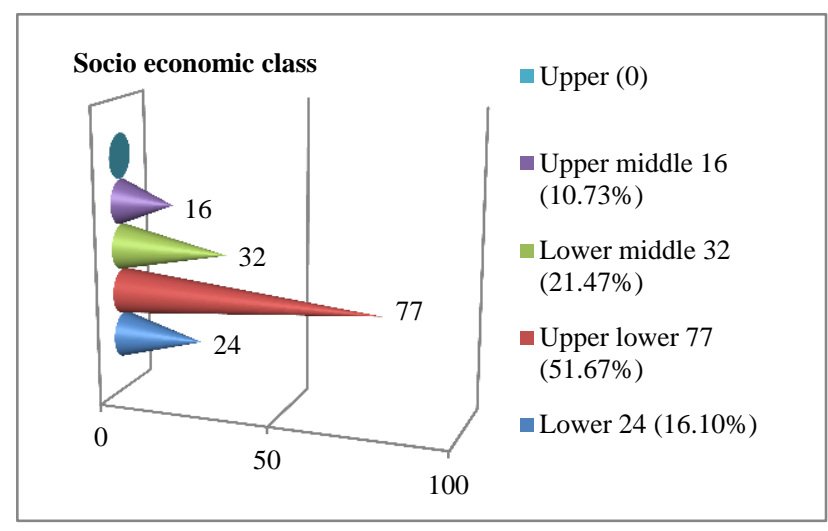

Figure 4: Distribution of study subjects based on Modified-Kuppuswamy's socio economic classification $2015(n=149)$.

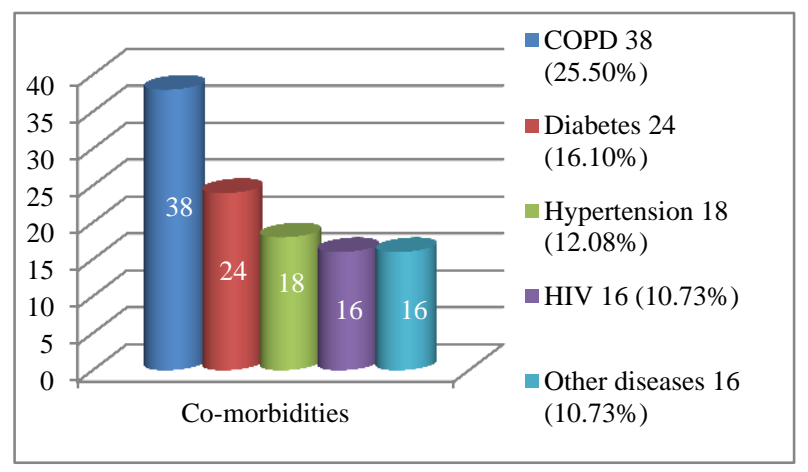

Figure 5: Distribution of study subjects based on the presence of co-morbidities $(n=149)$.

Others diseases included thyroid disorder (10) or coronary artery disease (3), epilepsy (2) or carcinoma (1), a few participants had multiple responses for the co-morbidities.

The relationship between factors and treatment outcome are depicted from Tables 2-4. The treatment outcomes are categorised as cured and other treatment outcomes. The study variables under each category of adherence factors are grouped as group $\mathrm{E}$ and $\mathrm{O}$ and their epidemiological association is assessed as incidence of cure rates in each group as Ie and Io, Risk-ratio or Relative risk (RR), risk difference or attributable risk, attributable fraction in exposed (AFe) are calculated. The statistical association is calculated by using Chi-square test and based on Chisquare value, $\mathrm{p}$ value they are interpreted as $\mathrm{S}$ for significant $(p<0.05)$ and NS for Not significant $(p>0.05)$.

Relationship between socio-demographic factors and treatment outcome is shown in Table 2. Those aging $>50$ years had a lower cure rate $(70.83 \%)$ with an attributable fraction of $14.07 \%$, illiterates had a lower cure rate $(70 \%)$ with an attributable fraction of $17.06 \%$, males had a lower cure rate $(77.57 \%$ ) with an attributable fraction of $11.94 \%$, Hindus had a lower cure rate $(76.66 \%)$ and an attributable fraction of $20.60 \%$, patients of lower socioeconomic class (upper-lower and lower class) had a lower cure rate $(74.25 \%)$ with an attributable fraction of $20.8 \%$. By applying the chi square test literacy status, religion and socio-economic class showed statistical significance on TB treatment outcome.

Relationship between adherence factors (patient related factors-a) and treatment outcome is shown in Table 3. The patient's knowledge was assessed based on their awareness about TB, its mode of spread, its curability, treatment duration and importance of completing the TB Treatment. The scoring was 1 for correct and 0 for incorrect answers the scores varied between 0 and 5 . Further total scores were categorised as poor for 0,1 and 2 score's and good for 3, 4 and 5 score's. A poor patient knowledge on TB treatment had a lower cure rate $(65.85 \%)$ with an attributable fraction of $23.52 \%$. Patients who did not believe that the TB treatment can cure the disease had a lower cure rate $(75.8 \%)$ with an attributable fraction of $9.65 \%$. Patients unwilling to continue treatment with improvement of symptoms had a lower cure rate $(75 \%)$ with an attributable fraction of $8.59 \%$. By applying Chi-square test poor patient knowledge on TB showed statistical significance on the TB treatment outcome.

Relationship between adherence factors (patient related factors-b) and treatment outcome is shown in Table 3. Smokers had a lesser cure rate $(73.25 \%)$ and smoking has an attributable fraction of $19.03 \%$. Alcoholics had a lesser cure rate $(67.27 \%)$ and alcohol intake has an attributable fraction of $23.80 \%$. Diabetics had a lesser cure rate $(75 \%)$ and diabetic status has an attributable fraction of $8.08 \%$. Hypertensive's had a lesser cure rate $(77.77 \%)$ and hypertensive status has an attributable fraction of $3.88 \%$. Patients with COPD had a lesser cure rate $(71.05 \%)$ with an attributable fraction of $15.19 \%$. Patients with HIV had a lesser cure rate $(50 \%)$ and an attributable fraction of $40.61 \%$. By applying Chi-square test smoking, alcohol intake, HIV status showed statistical significance on TB treatment outcome.

Relationship between adherence factors (health care related factors) and treatment outcome is depicted in 
Table 4. Patients who were unsatisfied with the available TB treatment health services had a lower cure rate $(63.63 \%)$ and an attributable fraction of $23.75 \%$. Patients who did not have appropriate access to DOTS center or provider had a lower cure rate $(70.96 \%)$ and an attributable fraction of $14.55 \%$.

Table 2: Relationship between socio-demographic factors and treatment outcome.

\begin{tabular}{|c|c|c|c|c|c|c|c|c|}
\hline \multirow[b]{2}{*}{$\begin{array}{l}\text { Study } \\
\text { variables }\end{array}$} & \multicolumn{2}{|c|}{ Treatment Outcome } & \multirow[b]{2}{*}{$\begin{array}{l}\text { Cure } \\
\text { rate } \\
(\%)\end{array}$} & \multirow[b]{2}{*}{$\begin{array}{l}\text { Risk } \\
\text { ratio } \\
\text { (relative } \\
\text { risk)* } \\
\quad I_{e} / I_{O}\end{array}$} & \multirow[b]{2}{*}{$\begin{array}{l}\text { Risk } \\
\text { difference or } \\
\text { attributable } \\
\text { risk } \\
\qquad I_{e}-I_{O}\end{array}$} & \multirow{2}{*}{$\begin{array}{l}\text { Attributabl } \\
\text { e fraction in } \\
\text { exposed } * * \\
\frac{I_{e}-I_{O}}{I_{o}} \times 100 \\
(\text { in } \%)\end{array}$} & \multirow[b]{2}{*}{$\begin{array}{l}\text { Chi- } \\
\text { square } \\
\text { value }\end{array}$} & \multirow[b]{2}{*}{ P value } \\
\hline & $\begin{array}{l}\text { Cured } \\
(n=120)\end{array}$ & $\begin{array}{l}\text { Other } \\
\text { treatment } \\
\text { outcomes } \\
(n=29)\end{array}$ & & & & & & \\
\hline \multicolumn{9}{|l|}{ Age (in years) } \\
\hline$>50_{\mathrm{e}(24)}$ & 17 & 7 & 70.83 & \multirow{2}{*}{0.85} & \multirow{2}{*}{11.6} & \multirow{2}{*}{14.07} & \multirow{2}{*}{$\begin{array}{l}1.72 \\
d f=1\end{array}$} & \multirow{2}{*}{$\begin{array}{l}0.189 \\
\text { (NS) }\end{array}$} \\
\hline$<50_{\mathrm{o}(125)}$ & 103 & 22 & 82.4 & & & & & \\
\hline \multicolumn{9}{|l|}{ Literacy status } \\
\hline Illiterate $_{\mathrm{e}(40)}$ & 28 & 12 & 70 & \multirow{2}{*}{0.82} & \multirow{2}{*}{14.4} & \multirow{2}{*}{17.06} & \multirow{2}{*}{$\begin{array}{l}3.87 \\
\mathrm{df}=1\end{array}$} & \multirow{2}{*}{$\begin{array}{l}0.049 \\
(\mathrm{~S})\end{array}$} \\
\hline Literate $_{o(109)}$ & 92 & 17 & 84.4 & & & & & \\
\hline \multicolumn{9}{|l|}{ Sex } \\
\hline Male $_{\text {e (107) }}$ & 83 & 24 & 77.57 & \multirow{2}{*}{0.88} & \multirow{2}{*}{10.52} & \multirow{2}{*}{11.94} & \multirow{2}{*}{$\begin{array}{l}2.13 \\
\mathrm{df}=1\end{array}$} & \multirow{2}{*}{$\begin{array}{l}0.144 \\
(\mathrm{NS})\end{array}$} \\
\hline Female $_{\mathrm{o}(42)}$ & 37 & 5 & 88.09 & & & & & \\
\hline \multicolumn{9}{|l|}{ Religion } \\
\hline Hindu $_{\mathrm{e}(120)}$ & 92 & 28 & 76.66 & \multirow[b]{2}{*}{0.79} & \multirow[b]{2}{*}{19.89} & \multirow[b]{2}{*}{20.60} & \multirow[b]{2}{*}{$\begin{array}{l}5.89 \\
\mathrm{df}=1\end{array}$} & \multirow[b]{2}{*}{$\begin{array}{l}0.015 \\
(\mathrm{~S})\end{array}$} \\
\hline $\begin{array}{l}\text { Muslim and } \\
\text { Christian }_{0}(29)\end{array}$ & 28 & 1 & 96.55 & & & & & \\
\hline \multicolumn{9}{|c|}{ Socio economic class } \\
\hline $\begin{array}{l}\text { Lower class and } \\
\text { upper lower }_{\mathrm{e}(101)}\end{array}$ & 75 & 26 & 74.25 & \multirow{2}{*}{0.79} & \multirow{2}{*}{19.5} & \multirow{2}{*}{20.8} & 7.89 & 0.004 \\
\hline $\begin{array}{l}\text { Lower middle and } \\
\text { upper middle } \\
\text { o (48) }\end{array}$ & 45 & 3 & 93.75 & & & & $\mathrm{df}=1$ & $(\mathrm{~S})$ \\
\hline
\end{tabular}

*: $\mathrm{RR}<1$ indicates that exposure reduces the cure rates; $* *: \mathrm{AFe}$ is the $\%$ of reduced cure rate in the exposed that is attributed to the exposure.

Table 3: Relationship between adherence factors and treatment outcome patient related factors (a and b).

\begin{tabular}{|c|c|c|c|c|c|c|c|c|}
\hline \multirow[b]{2}{*}{$\begin{array}{l}\text { Study } \\
\text { variables }\end{array}$} & \multicolumn{2}{|c|}{ Treatment outcome } & \multirow[b]{2}{*}{$\begin{array}{l}\text { Cure } \\
\text { rate } \\
(\%)\end{array}$} & \multirow[b]{2}{*}{$\begin{array}{l}\text { Risk ratio } \\
\text { (relative } \\
\text { risk)* } \\
\mathbf{I}_{\mathrm{e}} / \mathbf{I}_{0}\end{array}$} & \multirow[b]{2}{*}{$\begin{array}{l}\text { Risk } \\
\text { Difference or } \\
\text { attributable } \\
\text { risk } I_{e}-I_{0}\end{array}$} & \multirow[b]{2}{*}{$\begin{array}{l}\text { Attributable } \\
\text { fraction in } \\
\text { exposed } * * \\
\frac{I_{e}-I_{O}}{I_{o}} \times 100 \\
\text { (in } \% \text { ) }\end{array}$} & \multirow[b]{2}{*}{$\begin{array}{l}\text { Chi- } \\
\text { square } \\
\text { value }\end{array}$} & \multirow[b]{2}{*}{ P value } \\
\hline & $\begin{array}{l}\text { Cured } \\
(n=120)\end{array}$ & $\begin{array}{l}\text { Other } \\
\text { treatment } \\
\text { outcomes } \\
(n=29)\end{array}$ & & & & & & \\
\hline \multicolumn{9}{|c|}{ Patients knowledge about TB treatment } \\
\hline Poor $_{\mathrm{e}(41)}$ & 27 & 14 & 65.85 & \multirow{2}{*}{0.76} & \multirow{2}{*}{20.26} & \multirow{2}{*}{23.52} & \multirow{2}{*}{$\begin{array}{l}7.78 \\
\mathrm{df}=1\end{array}$} & \multirow{2}{*}{$\begin{array}{l}0.0053 \\
(\mathrm{~S})\end{array}$} \\
\hline $\operatorname{Good}_{\text {o(108) }}$ & 93 & 15 & 86.11 & & & & & \\
\hline \multicolumn{9}{|c|}{ Patients belief that the TB treatment can cure their disease } \\
\hline $\mathrm{No}_{\mathrm{e}(62)}$ & 47 & 15 & 75.8 & \multirow[b]{2}{*}{0.9} & \multirow{2}{*}{8.1} & \multirow{2}{*}{9.65} & \multirow{2}{*}{$\begin{array}{l}1.52 \\
\mathrm{df}=1\end{array}$} & \multirow{2}{*}{$\begin{array}{l}0.21 \\
(\mathrm{NS})\end{array}$} \\
\hline $\mathrm{Yes}_{\mathrm{o}(87)}$ & 73 & 14 & 83.90 & & & & & \\
\hline \multicolumn{9}{|c|}{ Patients willingness to continue treatment even after the symptoms subsides } \\
\hline Not willing $_{\mathrm{e}(32)}$ & 24 & 8 & 75 & \multirow{2}{*}{0.91} & \multirow{2}{*}{7.05} & \multirow{2}{*}{8.59} & \multirow{2}{*}{$\begin{array}{l}0.8 \\
\mathrm{df}=1\end{array}$} & \multirow{2}{*}{$\begin{array}{l}0.37 \\
(\mathrm{NS})\end{array}$} \\
\hline $\mathrm{Yes}_{\mathrm{o}(117)}$ & 96 & 21 & 82.05 & & & & & \\
\hline \multicolumn{9}{|c|}{ Smoker (currently smoker or who had smoked any form of tobacco for >1 year) } \\
\hline $\mathrm{Yes}_{\mathrm{e}(86)}$ & 63 & 23 & 73.25 & \multirow{2}{*}{0.80} & \multirow{2}{*}{17.22} & \multirow{2}{*}{19.03} & \multirow{2}{*}{$\begin{array}{l}6.88 \\
d f=1\end{array}$} & \multirow{2}{*}{$\begin{array}{l}0.008 \\
(\mathrm{~S})\end{array}$} \\
\hline $\mathrm{No}_{\mathrm{o}(63)}$ & 57 & 6 & 90.47 & & & & & \\
\hline \multicolumn{9}{|c|}{ Alcoholic (currently alcoholic or who had consumed alcohol for >1 year) } \\
\hline $\mathrm{Yes}_{\mathrm{e}(55)}$ & 37 & 18 & 67.27 & \multirow{2}{*}{0.76} & \multirow{2}{*}{21.02} & 2380 & 9.79 & 0.001 \\
\hline $\mathrm{No}_{0}(94)$ & 83 & 11 & 88.29 & & & 23.80 & $\mathrm{df}=1$ & $(\mathrm{~S})$ \\
\hline Diabetic & & & & & & & & \\
\hline $\mathrm{Yes}_{\mathrm{e}(24)}$ & 18 & 6 & 75 & & & 8.08 & 0.56 & 0.45 \\
\hline $\mathrm{No}_{0}(125)$ & 102 & 23 & 81.6 & 0.91 & 6.6 & & $\mathrm{df}=1$ & $(\mathrm{NS})$ \\
\hline
\end{tabular}




\begin{tabular}{|c|c|c|c|c|c|c|c|c|}
\hline \multirow[b]{2}{*}{$\begin{array}{l}\text { Study } \\
\text { variables }\end{array}$} & \multicolumn{2}{|c|}{ Treatment outcome } & \multirow[b]{2}{*}{$\begin{array}{l}\text { Cure } \\
\text { rate } \\
(\%)\end{array}$} & \multirow[b]{2}{*}{$\begin{array}{l}\text { Risk ratio } \\
\text { (relative } \\
\text { risk)* } \\
\mathrm{I}_{\mathrm{e}} / \mathbf{I}_{0}\end{array}$} & \multirow[b]{2}{*}{$\begin{array}{l}\text { Risk } \\
\text { difference or } \\
\text { attributable } \\
\text { risk } \mathrm{I}_{\mathrm{e}}-\mathrm{I}_{\mathbf{0}}\end{array}$} & \multirow[b]{2}{*}{$\begin{array}{l}\text { Attributable } \\
\text { fraction in } \\
\text { exposed } * * \\
\frac{I_{e}-I_{o}}{I_{o}} \times 100 \\
\text { (in } \% \text { ) }\end{array}$} & \multirow[b]{2}{*}{$\begin{array}{l}\text { Chi- } \\
\text { square } \\
\text { value }\end{array}$} & \multirow[b]{2}{*}{ P value } \\
\hline & $\begin{array}{l}\text { Cured } \\
\text { (120) }\end{array}$ & $\begin{array}{l}\text { Other } \\
\text { treatment } \\
\text { outcomes } \\
(29)\end{array}$ & & & & & & \\
\hline \multicolumn{9}{|c|}{ Hypertensive } \\
\hline $\mathrm{Yes}_{\mathrm{e}(18)}$ & 14 & 4 & 77.77 & \multirow{2}{*}{0.96} & \multirow{2}{*}{3.14} & \multirow{2}{*}{3.88} & \multirow{2}{*}{$\begin{array}{l}0.1 \\
\mathrm{df}=1\end{array}$} & \multirow{2}{*}{$\begin{array}{l}0.75 \\
(\mathrm{NS})\end{array}$} \\
\hline $\mathrm{No}_{\mathrm{o}(131)}$ & 106 & 25 & 80.91 & & & & & \\
\hline \multicolumn{9}{|c|}{ Chronic obstructive pulmonary disease (COPD) } \\
\hline Yes $_{\text {e (38) }}$ & 27 & 11 & 71.05 & \multirow{2}{*}{0.84} & \multirow{2}{*}{12.73} & \multirow{2}{*}{15.19} & \multirow{2}{*}{$\begin{array}{l}2.93 \\
\mathrm{df}=1\end{array}$} & \multirow{2}{*}{$\begin{array}{l}0.086 \\
\text { (NS) }\end{array}$} \\
\hline $\mathrm{No}_{\mathrm{o}(111)}$ & 93 & 18 & 83.78 & & & & & \\
\hline \multicolumn{9}{|c|}{ HIV status } \\
\hline $\mathrm{Yes}_{\mathrm{e}(16)}$ & 8 & 8 & 50 & \multirow{2}{*}{0.59} & \multirow{2}{*}{34.2} & \multirow{2}{*}{40.61} & \multirow{2}{*}{$\begin{array}{l}10.66 \\
\mathrm{df}=1\end{array}$} & \multirow{2}{*}{$\begin{array}{l}0.0011 \\
(\mathrm{~S})\end{array}$} \\
\hline $\mathrm{No}_{\mathrm{o}(133)}$ & 112 & 21 & 84.2 & & & & & \\
\hline
\end{tabular}

*: RR<1 indicates that exposure reduces the cure rates; **: AFe is the $\%$ of reduced cure rate in the exposed that is attributed to the exposure.

Table 4: Relationship between adherence factors and treatment outcome: health care related factors and social factors.

\begin{tabular}{|c|c|c|c|c|c|c|c|c|}
\hline \multirow[b]{2}{*}{$\begin{array}{l}\text { Study } \\
\text { variables }\end{array}$} & \multicolumn{2}{|c|}{ Treatment outcome } & \multirow[b]{2}{*}{$\begin{array}{l}\text { Cure } \\
\text { rate } \\
(\%)\end{array}$} & \multirow[b]{2}{*}{$\begin{array}{l}\text { Risk } \\
\text { ratio } \\
\text { (relative } \\
\text { risk)* } \\
\quad I_{e} / I_{O}\end{array}$} & \multirow{2}{*}{$\begin{array}{l}\text { Risk } \\
\text { difference } \\
\text { or } \\
\text { attribute } \\
\text { able risk } \\
\qquad I_{e}-I_{0} \\
\end{array}$} & \multirow{2}{*}{$\begin{array}{l}\text { Attributable } \\
\text { fraction in } \\
\text { exposed } * * \\
\frac{I_{e}-I_{O}}{I_{O}} \times 100 \\
(\text { in } \%)\end{array}$} & \multirow[b]{2}{*}{$\begin{array}{l}\text { Chi- } \\
\text { square } \\
\text { value }\end{array}$} & \multirow[b]{2}{*}{$\begin{array}{l}P \\
\text { value }\end{array}$} \\
\hline & $\begin{array}{l}\text { Cured } \\
(\mathbf{1 2 0})\end{array}$ & $\begin{array}{l}\text { Other } \\
\text { treatment } \\
\text { outcomes } \\
\text { (29) }\end{array}$ & & & & & & \\
\hline \multicolumn{9}{|c|}{ Health care related factors } \\
\hline \multicolumn{9}{|c|}{ Patients response for availability of TB treatment health services } \\
\hline Unsatisfactory $_{\mathrm{e}(22)}$ & 14 & 8 & 63.63 & \multirow{2}{*}{0.76} & \multirow{2}{*}{19.83} & \multirow{2}{*}{23.75} & \multirow{2}{*}{$\begin{array}{l}4.7 \\
d f=1\end{array}$} & \multirow{2}{*}{$\begin{array}{l}0.030 \\
(\mathrm{~S})\end{array}$} \\
\hline Satisfactory $_{0}(127)$ & 106 & 21 & 83.46 & & & & & \\
\hline \multicolumn{9}{|c|}{ Patients response for access to DOTS center or provider } \\
\hline Not accessible $_{\mathrm{e}(31)}$ & 22 & 9 & 70.96 & \multirow{2}{*}{0.85} & \multirow{2}{*}{12.09} & \multirow{2}{*}{14.55} & \multirow{2}{*}{$\begin{array}{l}2.29 \\
d f=1\end{array}$} & \multirow{2}{*}{$\begin{array}{l}0.130 \\
(\mathrm{NS})\end{array}$} \\
\hline Accessible $_{o(118)}$ & 98 & 20 & 83.05 & & & & & \\
\hline \multicolumn{9}{|c|}{ Patients response about contact timings with the health services } \\
\hline Unsatisfactory $_{\mathrm{e}(44)}$ & 33 & 11 & 75 & \multirow{2}{*}{0.90} & \multirow{2}{*}{7.85} & \multirow{2}{*}{9.47} & \multirow{2}{*}{$\begin{array}{l}1.22 \\
\mathrm{df}=1\end{array}$} & \multirow{2}{*}{$\begin{array}{l}0.269 \\
(\mathrm{NS})\end{array}$} \\
\hline Satisfactory $_{o}(105)$ & 87 & 18 & 82.85 & & & & & \\
\hline \multicolumn{9}{|l|}{ Social factors } \\
\hline \multicolumn{9}{|c|}{ Encouragement from family members to continue treatment } \\
\hline $\mathrm{No}_{\mathrm{e}(32)}$ & 24 & 8 & 75.0 & \multirow{2}{*}{0.91} & \multirow{2}{*}{7.05} & \multirow{2}{*}{8.59} & \multirow{2}{*}{$\begin{array}{l}0.8 \\
\mathrm{df}=1\end{array}$} & \multirow{2}{*}{$\begin{array}{l}0.371 \\
(\mathrm{NS})\end{array}$} \\
\hline $\mathrm{Yes}_{\mathrm{o}(117)}$ & 96 & 21 & 82.05 & & & & & \\
\hline Patients marital status & & & & & & & & \\
\hline Other marital status $_{\mathrm{e}(55)}$ & 39 & 16 & 70.9 & 082 & 1527 & 1777 & 5.16 & 0.023 \\
\hline Married $_{\mathrm{o}(94)}$ & 81 & 13 & 86.17 & 0.82 & 15.21 & 11.12 & $\mathrm{df}=1$ & $(\mathrm{~S})$ \\
\hline $\begin{array}{l}\text { Patients response to ha } \\
\text { friends) }\end{array}$ & experi & ed some fo & 1 of sti & a due & (being & led or isolat & by famil & \\
\hline $\mathrm{No}_{\mathrm{e}(111)}$ & 89 & 22 & 80.18 & 098 & 139 & 169 & 0.04 & 0.84 \\
\hline $\mathrm{Yes}_{\mathrm{o}(38)}$ & 31 & 7 & 81.57 & 0.98 & 1.39 & 1.69 & $\mathrm{df}=1$ & (NS) \\
\hline
\end{tabular}

*: RR $<1$ indicates that exposure reduces the cure rates; **: AFe is the $\%$ of reduced cure rate in the exposed that is attributed to the exposure.

Patients who were unsatisfied with the contact timings of the health facility had a lower cure rate $(75 \%)$ and an attributable fraction of $9.47 \%$. By applying Chi-square test availability of TB treatment health services showed statistical significance on the treatment outcome.

Relationship between adherence factors (social factors) with treatment outcome is shown in Table 4. Patients who had no encouragement from family members to continue treatment had a lower cure rate $(75 \%)$ and an attributable fraction of $8.59 \%$. Patients with other marital status had a lower cure rate $(70.9 \%)$ and an attributable fraction of $17.72 \%$. Patients who didn't experience stigma due to TB had a lower cure rate of $80.18 \%$ and an attributable fraction of $1.69 \%$. By applying chi-square test patients marital status showed statistical significance on $\mathrm{TB}$ treatment outcome. 


\section{DISCUSSION}

A prospective study assessing various factors of adherence to treatment on TB-treatment outcome among NSSP pulmonary TB patients showed a male: female ratio of 2.5:1 and of ages 18-60 years, similar to a study done by Gajbhare et al were majority of the TB patients were males and belonged to $15-44$ years age. ${ }^{10}$ This distribution among males and economically productive age group can have an impact on the economic status of the family.

Majority of the study participants in this study belonged to upper lower class similar to a study done by Sadana et al were majority were of upper lower class indicating a major distribution of TB in lower socio economic stratum (SES). ${ }^{11}$

\section{Socio-demographic factors}

Age $>50$ years, illiteracy, male gender and lower SES and Hindu religion attributed to lesser cure rates among the study subjects, of these factors further illiteracy, Hindu religion and Lower SES showed a statistically significant association with TB treatment outcome. In a study by Sadana et al statistically significant association was seen for gender, literacy status $(p=0.03)$ but SES $(p=0.639)$ showed no statistical significance with the treatment outcome. ${ }^{11}$

\section{Adherence to treatment factors}

\section{Patient related factors}

Patient's- poor knowledge about TB, disbelief in TB treatment, unwillingness to continue treatment with subsiding symptoms, habits like smoking and alcohol intake, presence of diabetes, hypertension, COPD and HIV attributed to lesser cure rates, Of these factors poor patient's knowledge on TB, smoking, alcohol intake and HIV positive status showed a statistically significant association with TB treatment outcome. A study done by Sunil et al showed significant association between smoking and alcoholism with treatment outcome. ${ }^{12}$ A systematic review also shows an increased risk of TB disease and mortality among smokers. ${ }^{13}$ According to a study done by Vijay et al there was a significantly higher proportion of treatment failures among TB-DM patients than TB non-DM patients $(\mathrm{p}=0.04) .{ }^{14} \mathrm{~A}$ study done by Vijay et al showed a lesser treatment success rate (62\%) among TBHIV patients in comparison with TB non-HIV patients. ${ }^{15}$

\section{Health care related factors}

Patient's unsatisfactory response for availability of TB treatment, contact timings with health facility and accessibility to the DOTS center or provider attributed to lower cure rates. Of these factors patient's unsatisfaction with the TB treatment services showed a statistically significant association with TB treatment outcome and the most common reason for unsatisfaction was inadequate on-time care to TB treatment side effects.

\section{Social factors}

No encouragement from family members to continue treatment, other marital status (excluding married) and no stigma due to TB attributed to lower cure rates. Of these further patients other marital status showed a statistically significant association with TB treatment outcome.

\section{CONCLUSION}

Among the factors showing statistically significant association with TB-treatment outcome, by improving patient's knowledge on TB treatment, encouraging them to quit smoking and alcohol intake, appropriate TB-HIV treatment and improvement in health services as specified in the recommendations could contribute to better cure rates.

\section{Recommendations}

Among the factors showing association with treatment outcome the ones which can be addressed are poor patients knowledge on TB-treatment can be addressed by appropriate patient education through IEC materials, counselling at the DOTS center regarding treatment duration and regimen, complications the patients might have to face if the treatment is not taken completely like retreatment, drug resistance at different levels which is further associated with longer treatment durations and complex regimens, this could further reinforce the patients to adhere to the treatment. Habits like smoking and alcohol intake can be addressed by supportive counselling to quit such habits. Patient's un-satisfaction with the TB treatment services due to lack of on-time care to the side effects of TB drugs, ART drugs (those with TB-HIV) can be addressed by appropriate strengthening of referral and clinical services, motivational support and nutritional advice to the patients by effective counselling. The health care workers need to be educated regarding the same to improve the patient provider relationship. 38 patients had experienced social stigma due to TB, although it did not show statistical association with treatment outcome. In order to counter the social stigma and myths associated with the disease were awareness can be created about TB in general population as a curable disease through posters and mass media usage, an efficient social support system can be created by providing motivational services from the patients cured of $\mathrm{TB}$, to the patients and their family members currently under treatment.

\section{ACKNOWLEDGEMENTS}

Our sincere thanks to Dr Nagaraj (Prof and HOD Department of Pulmonology BMCRI) and Dr. Arundati Das (DTO, Bengaluru) who permitted for the study, 
Medical officer's, staff and study subjects at the DOTS centre's for their co-operation during the study.

Funding: RNTCP (BBMP TB unit)

Conflict of interest: None declared

Ethical approval: The study was approved by the Institutional Ethics Committee

\section{REFERENCES}

1. Park K. Park's textbook of preventive and social medicine. 23rd ed. Jabalpur, India: M/s Banarsidas Bhanot; 2015;176-202:427-30.

2. WHO. Tuberculosis fact sheets, 2016 March; Available at: http://www.who.int/mediacentre/factsh eets/fs104/en/. Accessed on 9 August 2016.

3. The Global Plan to End TB 2016-2020, 2015. Available at: http://www.stoptb.org/global/plan/plan 2/. Accessed on 27 August 2016.

4. Gupta I, Guin P. Communicable diseases in SouthEast Asia region of the World Health Organisation: towards a more effective response. Bull World Health Organ. 2010;88:199-205.

5. RNTCP Technical and Operational Guidelines for Tuberculosis Control in India, 2016. Available at: http://www.tbcindia.nic.in/index 1.php?lang=1 andle vel $=2$ andsublinkid $=4573$ andlid $=3177$. Accessed on 4 September 2016.

6. Global Tuberculosis report 2015, 2015. Available at: http://www.who.int/tb/publications/global_ report/gtbr2015_executive_summary.pdf. Accessed on 28 August 2016.

7. Central TB Division. TB INDIA 2016 Annual status report, 2016. Available at http://www.tbcindia. nic.in/WriteReadData/1892s/3608495828TB\%20Ind ia\%202016_Part1.pdf. Accessed 8 August 2016.

8. WHO. Adherence to long-term therapies, 2003. Available at: http://apps.who.int/iris/bitstream/ 10665/42682/1/9241545992.pdf. Accessed on 27 August 2016.
9. Adherence to treatment for latent tuberculosis infection: a manual for health care providers, 2005. Available at: http://dph.georgia.gov/sites/dph.georgI a.gov/files/TBLTBI_TreatmentManuaHarlem.pdf. Accessed on 12 August 2016.

10. Gajbhare D, Bedre R, Solanki H. A study of sociodemographic profile and treatment outcome of tuberculosis patients in an urban slum of Mumbai. Indian J Basic Appl Med Res. 2014;4(1):50-7.

11. Sadana P, Singh T, Deepti SS. Socio demographic factors affecting the treatment outcome in patients of tuberculosis. Nat $\mathrm{J}$ Community Med. 2015;6(4):609-13.

12. Sunil MS, Rahul RB. Socio-demographic profile of patients registered under revised national tuberculosis control programme in Pune city. Int J Biomed Res. 2014;5(3):168-71.

13. Detels R, Gulliford M, Karim Q, Tan C. Oxford Textbook of Global Public Health. 6th ed. Great Clarendon Street, Oxford: Oxford University Press; 2016: 1144-1160.

14. Vijay V, Vigneswari A, Selvan K, Satyavani K, Rajeswari R, Kapur A. Effect of diabetes on treatment outcome of smear-positive pulmonary tuberculosis- a report from South India. J Diabet Complicat. 2014;28(2):162-5.

15. Vijay S, Kumar P, Chauhan LS, Narayan Rao SV, Vaidyanathan P. Treatment outcome and mortality at one and half year follow-up of HIV infected TB Patients Under TB Control Programme in a District of South India. PLoS ONE. 2011;6(7):e21008.

Cite this article as: Ramya MS, Jadhav J, Ranganath TS. A study to assess the association of various factors of adherence to treatment on the tuberculosis treatment outcome among new sputum smear positive pulmonary tuberculosis patients under revised national tuberculosis control programme in Bengaluru area. Int J Community Med Public Health 2019;6: 4344-51. 